\title{
Enhanced quality of service support for triple play services in IEEE 802.11 WLANs
}

Yahya Javed $^{1 *}$, Adeel Baig $^{1,2}$ and Maajid Maqbool ${ }^{1}$

\begin{abstract}
Wi-Fi or wireless local area networks (WLANs) are among the most popular wireless internet access technologies used. The major challenge faced by WLANs is the provision of quality of service (QoS) for real-time applications at high congestion periods. IEEE 802.11e draft presents the only comprehensive QoS infrastructure for WLANs which proposes enhanced distributed channel access (EDCA) that propounds the prioritization of medium access for different traffic classes. EDCA while making perceptible improvements for real-time applications neglects non-real-time applications by allocating their share of the bandwidth to the former in an inefficient manner. In our study, we have found that there are considerable design improvements possible for IEEE 802.11e-based QoS propositions. This paper proposes a novel medium access and transmission mechanism for IEEE 802.11 WLANs that is specifically designed to ensure QoS for triple play services. Based on our study regarding the traffic characteristics of triple play services, we propose a mechanism that adaptively uses the medium access and transmission parameters according to the traffic characteristics of the applications, for better utilization of the available bandwidth. The mechanism also proffers higher medium access priority to the access point as compared to the stations in order to cope with the issue of uplink/downlink traffic asymmetry. Simulation-based analysis of the proposed mechanism compares its performance with EDCA. The proposed mechanism offers promising results in terms of packet loss, packet delay and throughput, and thus ensures QoS for voice, video and data transfer applications.
\end{abstract}

Keywords: Quality of service (QoS); IEEE 802.11e; Triple play services; Medium access control (MAC); Medium access priority; Traffic asymmetry

\section{Introduction}

Today, every internet user is familiar with the term Wi-Fi. Our homes, restaurants, airports and even trains, buses and commercial airplanes provide Wi-Fi connectivity. The large-scale use of smartphones and the popularity of voice chat applications have also made Wi-Fi a widely used network for communications. The major utility of Wi-Fi is cheap internet access and support for high data rates, but this explosive increase in Wi-Fi usage has its repercussions that a shared bandwidth (up to 600Mbps) is divided between all of the stations running different applications simultaneously due to which degradation in performance occurs especially for real-time applications. Therefore, it is required by the network to provide appropriate services to delay sensitive

\footnotetext{
* Correspondence: 09bicseyjaveed@seecs.edu.pk

${ }^{1}$ School of Electrical Engineering and Computer Science (SEECS), National University of Sciences and Technology (NUST), Sector H-12, Islamabad 44000, Pakistan

Full list of author information is available at the end of the article
}

applications and guaranteed required bandwidth to the triple play services of voice, video and internet access. The IEEE 802.11e draft presents the sole brief quality of service (QoS) framework for IEEE 802.11 wireless local area networks (WLANs) by introducing enhanced distributed channel access (EDCA) [1], which characterizes traffic into high and low priority traffic classes. EDCA implementations divide traffic into four major categories of voice, video, best effort and background traffic. Each traffic category gets different medium access priority with voice getting the highest, video second highest, best effort second least and background getting the least priority to access the medium. This medium access priority is enforced by awarding different contention window sizes to different traffic categories with highest priority traffic category getting the lowest contention window size and different sizes of arbitration inter-frame space (AIFS) with high priority traffic transmitting with shorter inter-frame space. To increase the transmission efficiency, EDCA uses transmission

\section{实}


opportunity (TXOP) limit, a definite time interval in which a station can transmit any number of frames with short inter-frame space (SIFS) without contention. The approach followed in EDCA makes significant performance enhancements for real-time applications, but at the same time neglects non-real-time applications. Moreover, the medium access and transmission parameters for different traffic categories do not take into account the traffic characteristics of the applications which result in bandwidth underutilization and unfair distribution of channel among different applications [2]. Use of AIFS and TXOP limit by EDCA in a non-adaptive manner for different applications having diverse traffic characteristics and behaviour result in notable performance degradation.

The proposed mechanism addresses the issues by (1) making the sizes of the contention windows and TXOP limit adaptive based on the traffic characteristics, (2) offering adaptive transmission style through manipulation of parameters like inter-frame spacing and frame aggregation and (3) making the medium access priority of the access point higher than that of the stations for most of the traffic types, to increase the overall downlink throughput as proposed in [3]. This paper introduces our proposed medium access and transmission scheme for WLANs and provides a simulation-based study and performance analysis of the proposed mechanism.

We start with a discussion on background and related works in Section 2, followed by a brief analysis on traffic characteristics of voice, video and data applications in Section 3. Section 4 explains the proposed mechanism while Section 5 presents the simulation setup. Section 6 compares the proposed mechanism with EDCA along with the analysis, and Section 7 provides discussion and performance evaluation of the proposed mechanism. Section 8 discusses some of the possible future direction of implementation while Section 9 concludes the paper.

\section{Background and related work}

There are two generic approaches for the provision of QoS: (1) integrated services and (2) differentiated services [4]. The differentiated services approach has been followed in IEEE 802.11 WLANs up till now which advocates priority-based QoS for different traffic types described in [1] and [5,6].

Integrated services involve end-to-end reservation mechanisms and are generically designed for wired networks, but some work has been initiated in this regard in the wireless domain also like in DARE, a distributed end-to-end reservation protocol that uses reservation techniques for QoS in wireless mesh networks [7]. The basic protocol followed by the integrated services approach is the Resource Reservation Protocol (RSVP) [8]. Reservation protocols require signaling of information between nodes and thus cause signaling overhead as in the MACA protocol [9], due to which they were unable to gain much popularity for wireless networks.

Differentiated services-based QoS support is more popular in wireless networks as it requires less processing at the nodes, no signaling overheads and a distributed framework. What essentially is missing in differentiated services-based QoS deployments is their adaptability in medium access and transmission for different traffic types according to their traffic characteristics. Moreover, current Wi-Fi deployments treat traffic at the uplink and the downlink with the same priority despite the fact that there exists a significant traffic asymmetry. To cope with the traffic asymmetry problem, Gupta et al. presented their access point only solution [3] that treats all of the traffic types in a similar manner but provides differentiated services to uplink and downlink traffic. Their proposed protocol (WiFox) resulted in overall throughput enhancement but what largely is missing in their work is the absence of traffic specific adaptability of the protocol for different traffic types.

Chen et al. in their recent work presented a highperformance distributed coordination function with QoS support (QHDCF) protocol [10], which proposes a probability-based medium access mechanism by making use of priorities and number of stations in different traffic classes. The protocol betters the identified problem of EDCA, that EDCA while providing higher medium access to high priority traffic classes causes starvation for low priority traffic classes. But the work does not address the issue of traffic asymmetry neither it takes into account the traffic behaviour for optimal utilization of the available bandwidth.

Some recent works on QoS for WLANs show the growing trend towards time division multiplex (TDM)based contention-free solutions. In [11], Shankar et al. proposed their modified wireless token network (MWTN) medium access scheme to avoid collisions in the first place by modifying point coordination function (PCF)-based wireless token network (WTN). While TDM-based medium access schemes have their advantages, there exists a genuine criticism on them that they cause bandwidth under-utilization and wastage.

Mishima et al. recently proposed a distributed coordination function (DCF)-based multi-user medium access control (MAC) protocol [12], in which the access point controls the medium access for different traffic categories based on their specific QoS requirements. While their proposed protocol promises better QoS support for those stations that have attained medium access, there is a great possibility for other contending stations of not being able to access the medium for longer periods which might lead to starvation. Moreover, there is also a great deal of processing required to be carried out at the 
access point which could lead to processing delays as well. Their proposed framework explicitly requires the fragmentation of each frame which could lead to performance setbacks for video streaming traffic. In [13], Bao et al. presented a similar approach in which resource allocation based on different QoS requirements is performed by converting specific QoS requirements into data rate requests by the stations and then the subsequent assignment of sub-channels based on these data rate requests. In addition to the aforementioned reservations regarding the approach, the conversion of specific QoS requirements to simple rate requests oversimplifies the issue as there are other QoS parameters that are to be taken care of for a brief QoS support than just the data rates. Furthermore, frequent exchange of rate requests and their subsequent replies introduces significant signaling overhead as discussed earlier in this section.

Sarret et al. lately attempted to address the much debated issue of fairness in IEEE 802.11e QoS propositions [14]. To offer more medium access opportunities to low priority traffic classes, they propose the Smart Aggregation framework that forms a MAC frame by aggregating packets belonging to different traffic categories. While their work promises better fairness for low priority traffic classes, there are some open issues as well. First, the aggregation of high priority traffic (real-time) packets with low priority traffic (non-real-time) packets could adversely affect the performance of real-time applications like video streaming as not all packets in the aggregated MAC frame are video streaming packets due to which packets left in the video transmission queue would be subjected to next MAC frame, necessitating the contention for medium access again which could lead to the addition of significant delay. Secondly, while the mechanism tries to ensure fairness among different traffic categories of the same station starvation could be caused for other stations even if they have higher priority traffic to transmit due to higher time required by the station occupying the medium to transmit the larger-sized aggregated frame in a multi-station WLAN environment.

Some recent work regarding provision of QoS in WLANs show the initiation of efforts regarding creation of adaptive medium access mechanisms based on IEEE 802.11e QoS propositions as an acknowledged way forward for the issue. Achary et al. in their work presented an approach that adaptively changes the contention window sizes of different traffic classes based on the medium access priorities and length of their transmission queues [15]. The major contribution of their proposed scheme is the avoidance of collisions between the packets from same traffic classes, but what their approach is unable to utilize fully is the traffic characteristic-based medium access adaptability involving other medium access and transmission parameters like TXOP limit and inter-frame spacing for comprehensive solution to the problem.

\section{Traffic characteristics}

In order to utilize the bandwidth in the most efficient manner, it is essential to understand the traffic characteristics and behaviour of the traffic generated by triple play applications. This section briefly describes the traffic characteristics of the applications widely used by triple play services.

\subsection{Voice/VOIP}

For voice over internet protocol (VOIP) traffic, the bit rate, frame interval and the time interval between frames depend upon the type of codec being used. The most popular codec used by VOIP applications is ITU G.729 [16] followed by ITU G.711 [17]. Telecom systems use adaptive multi-rate (AMR) codec [18]. The transport protocol used by all of the VOIP applications is realtime transport protocol (RTP) [19]. Statistics suggest that $93 \%$ of VOIP traffic use ITU G.729 codec while the remaining 7\% use ITU G.711 among the ITU standard codecs [20]. Summary of VOIP traffic characteristics corresponding to different codecs is mentioned in Table 1.

Table 1 shows that VOIP traffic is bursty in nature with small data bursts and large silent intervals. VOIP also requires constant bit rate for smooth working of its applications. Moreover, the high time-critical nature of VOIP makes it more vulnerable to packet loss and packet delay. Also, voice traffic has a symmetric traffic volume at the uplink and the downlink.

\subsection{Video streaming/IPTV}

Video streaming traffic makes up to $25 \%$ to $40 \%$ of the overall internet traffic [21]. The traffic characteristics of the video streaming traffic depends upon the application (web browser) and the container (Flash [22], Silverlight [23] and HTML5 [24]). There are different streaming strategies used by the web sources of the video content. In this paper, we consider the most popular video streaming sources of Youtube and Netflix [25].

Video streaming of a particular video works in two phases: a buffering phase and a steady state phase. When the session is initiated, a stream of packets follows from the source server to fill the client's buffer. Once the buffer contains a certain amount of data, then the video playback begins even if the buffering phase is not over. After the buffering phase, the steady state phase is initiated in which there are ON-OFF cycles of data transfer. Youtube and Netflix use three kinds of streaming strategies: 
Table 1 Traffic characteristics corresponding to different VOIP codecs

\begin{tabular}{lcccc}
\hline Codec & $\begin{array}{l}\text { Bit rate } \\
\text { (kbps) }\end{array}$ & $\begin{array}{l}\text { Packet size } \\
\text { (bytes) }\end{array}$ & $\begin{array}{l}\text { Packet } \\
\text { interval }(\boldsymbol{\mu s})\end{array}$ & $\begin{array}{c}\text { Inter-packet } \\
\text { interval }(\mathbf{m s})\end{array}$ \\
\hline ITU G.711 & 64 & 284 & 2.27 & 20 \\
& & 364 & 2.91 & 30 \\
ITU G.729 & 6.4 & 134 & 1.07 & 10 \\
& 8 & 144 & 1.15 & 20 \\
& 11.4 & 154 & 1.23 & 30 \\
\hline
\end{tabular}

- No ON-OFF cycles

- Short ON-OFF cycles

- Long ON-OFF cycles

In no ON-OFF cycles, there is no steady phase and all of the data is transferred in the buffering phase while in short ON-OFF cycles, there is a steady state phase in which there is an $\mathrm{ON}$ period for $2.5 \mathrm{Mb}$ of data followed by an OFF period in which no data is transmitted by the streaming source. Long ON-OFF cycles streaming strategy is similar to short ON-OFF cycles except for the ON period which is for more than $2.5 \mathrm{Mb}$ of data [26].

The use of different streaming strategies change the traffic characteristics of video streaming traffic to some extent but video streaming traffic consists of back-to-back packets sent over a considerable amount of time no matter which streaming strategy is used. Video streaming traffic is asymmetric meaning its traffic volume is much larger at the downlink than at the uplink [3].

\subsection{Web-browsing}

Most of the internet traffic consists of the web-browsing traffic, and nowadays, many applications rely on the hypertext transfer protocol (HTTP) as their application layer protocol for web-browsing. HTTP has multiple versions among which HTTP 1.0 [27] and HTTP 1.1 [28] are the most popular. HTTP 1.0 can download back-to-back requested objects with one TCP connection per object but there is a limit to the number of connections and any left objects are retrieved from the web source after one of the ongoing connections terminate. HTTP 1.1 is similar to HTTP 1.0 except that it allows persistent connections and requests are pipelined. A webpage [29] is a hypertext document containing text of hypertext markup language (HTML) which links to other objects stored somewhere on another file or server. The hypertext document is called the main object while the linked objects are called in-line objects. A typical HTTP session consists of ON-OFF periods with ON periods corresponding to the period between the request and the retrieval of the last object. The OFF period is the silent interval when no data is transmitted [30].
All of the traffic models for web-browsing presented so far conform to the bursty and asymmetric nature of web-browsing traffic which shows that its traffic consists of bursts of data transfer periods followed by silent intervals with asymmetric uplink/downlink traffic volume [31].

\subsection{File-sharing}

File transfer protocol (FTP) is one of the internet's oldest application layer protocol used for file-sharing. Many simulators have built-in FTP traffic generation capability while many FTP traffic generators are available for use in test beds. The traffic pattern of FTP traffic is relatively predictable as compared to other traffic types mentioned in this paper. A typical FTP session involves the transmission of a number of packets. These packets are transmitted back-to-back while some of the packets are delayed due to the termination of a particular connection in a session, and most of them are transmitted with small-sized inter-packet intervals [32].

In short, FTP traffic consists of a stream of back-toback packets similar to video streaming traffic with a difference that it has no ON-OFF cycle mechanism for transmission rate control as in the case of video streaming, but FTP traffic is asymmetric like video streaming traffic [3].

\section{Proposed mechanism}

The proposed mechanism maximizes the utility of bandwidth resources and distributes the channel efficiently between stations running different applications while prioritizing medium access for time-critical applications. Furthermore, to increase the downlink throughput, the proposed mechanism grants the access point greater medium access priority as compared to the stations for most of the traffic categories. In addition to the classification of different traffic and their subsequent prioritization over each other, the proposed mechanism utilizes the traffic behaviour and selects an appropriate transmission style for the type of traffic that is about to be transmitted by the station or the access point. Figure 1 shows the architecture of the proposed mechanism while the following subsections discuss the system in detail.

\subsection{Traffic and station prioritization}

The proposed mechanism classifies traffic into four categories of voice, video, web-browsing and file-sharing. Table 2 gives the overall medium access priorities of the aforementioned traffic categories by positioning them in the range of 0 to 6 , with 0 representing the highest and 6 representing the lowest priority.

In Table 2, it can be seen that voice gets the highest priority with same medium access priority for access 


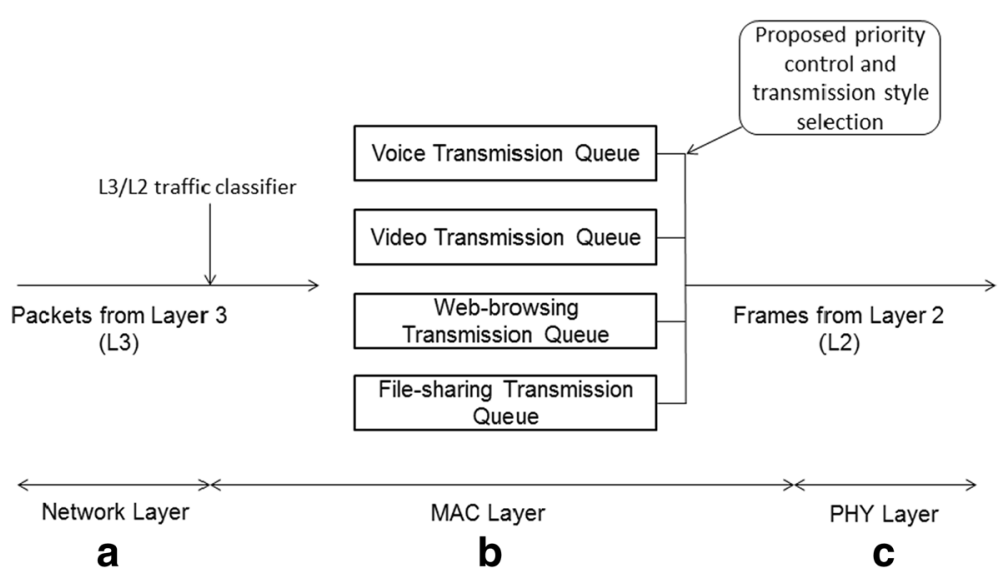

Figure 1 Architecture of the proposed mechanism. (a) Packet processing at the network layer. (b) Assignment of transmission queues (TxQ) and subsequent proposed priority control and frame transmission style selection at MAC layer. (c) Processing of datagrams at PHY layer.

point and the stations because for voice, similar amount of traffic is present at the uplink and the downlink. For video, access point gets higher priority than the stations as video traffic volume is higher at the downlink than that of the uplink, so allocating the medium to the stations equal to that of the access point in the video traffic case would waste the bandwidth. For web-browsing and file-sharing traffic categories, access point gets higher priority to access the medium than the stations in order to cope with the asymmetric behaviour of their traffic. The proposed mechanism allows traffic types like network control to access the medium with least priority similar to EDCA.

\subsection{Transmission styles}

Based on the traffic characteristics mentioned in Section 3 , the proposed mechanism subjects each traffic category to a different transmission style. For each transmission style, the transmission parameters like inter-frame space are different. The MAC frame aggregation mechanisms proposed in IEEE $802.11 \mathrm{n}$ standard are used by the system according to their efficiency for different categories of traffic [33]. Moreover, IEEE 802.11e block acknowledgment recommendations are upheld in the proposed scheme. The pseudo-code for transmission function of inter-frame space determination is given below along with the brief explanation of the process.

Table 2 Priority distribution for traffic categories

\begin{tabular}{lcc}
\hline Traffic category & Station priority & Access point priority \\
\hline Voice & 0 & 0 \\
Video & 2 & 1 \\
Web-browsing & 4 & 3 \\
Filesharing & 5 & 4 \\
Network control & 6 & 6
\end{tabular}

1. Voice: to accommodate the bursty nature of voice traffic, reduced inter-frame space (RIFS) introduced in the IEEE 802.11n standard is used by the proposed mechanism as RIFS is more suitable for traffic that has considerable inter-frame interval but require higher medium access priority [34]. No frame aggregation is used by the mechanism for voice data traffic.

2. Video: video traffic consists of a stream of packets so the proposed mechanism uses frame aggregation for the entire period of TXOP limit which means no inter-frame space between the frames.

3. Web-browsing: web-browsing traffic has a bursty nature (small data intervals and large silent intervals) so SIFS is used.

4. File-sharing: frame aggregation is used by the proposed mechanism for file-sharing traffic, for the entire TXOP limit period as file-sharing traffic consist of streams of back-to-back packets which are unordered. However, file-sharing traffic is insensitive to unordered packet delivery.

There are no individual acknowledgements for every frame transmitted by the nodes in the proposed scheme, but instead, there are block acknowledgements for every frame transmitted during the entire TXOP limit period.

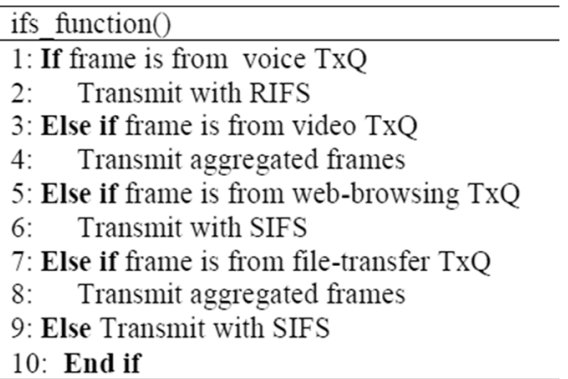




\subsection{Adaptive TXOP limit}

In order to increase throughput efficiency and to ensure fairness among different traffic applications, we propose adaptive TXOP limit for each application, so that the applications generating low priority traffic should not starve. The TXOP limit for each traffic type is different and mentioned in Table 3.

The TXOP limit values in Table 3 are written in a form to show the relativity of these values for different traffic types. Different combinations of numerical values were used when the proposed mechanism was simulated, but the relativity mentioned in Table 3 was maintained. Voice gets the least TXOP limit because it consists of short data bursts and long silent periods. Video gets highest TXOP limit as it has streams of packets, so that it could transmit as much of the stream as possible when the station acquires medium access. Web-browsing gets moderate TXOP limit because its traffic has bursty characteristics as well. File-sharing traffic gets a bit higher TXOP limit than web-browsing because it also has streams of packets, but has less priority than video traffic as file-sharing traffic is elastic (non-real-time) in nature.

\section{Test and simulation environment}

\subsection{Simulation setup}

The proposed medium access and transmission mechanism has been implemented using the open source simulation tool NS-2 [35]. We have used the TKN EDCA Model for NS-2 [36] for the EDCA simulations, but to simulate the proposed mechanism, we had to modify the TKN EDCA Model according to the propositions mentioned in Section 4 by (1) changing the medium access priorities for different traffic, (2) awarding higher medium access priority to the access point for some of the traffic, (3) associating different transmission styles with different traffic queues and (4) adaptively changing the TXOP limit for each traffic queue. By integration of the modified TKN EDCA Model with the 802.11 MAC layer of NS-2, the proposed mechanism was successfully implemented. Data samples were taken for simulation runtimes of up to $5,000 \mathrm{~s}$ during which most of the nodes try to transmit simultaneously, but there are silent intervals for some nodes as well. It was observed that steady state of the

\section{Table 3 TXOP limits for different traffic categories}

\begin{tabular}{ll}
\hline Traffic category & Relative TXOP limit \\
\hline Voice & Low \\
Video & Highest \\
Web-browsing & Moderate \\
File-sharing & Moderately high \\
Network control & Lowest \\
\hline
\end{tabular}

simulations was achieved for every simulation implying consistency in the results. The WLAN physical layer protocol used is IEEE $802.11 \mathrm{n}$. The simulation results were compiled by simulating six different network topologies with each simulation being run several times with different combination of access and transmission parameters. Figure 2 illustrates a model topology setup in consideration of a general WLAN environment. The traffic model used for each of the traffic types is according to their traffic characteristics mentioned in Section 3. Moreover, in order to view the complete picture and provide brief analysis, the performance evaluation is carried out for uplink and downlink of each traffic type separately. The simulations take into account the major QoS parameters of throughput, packet delay and packet loss for each station and the access point by precisely measuring the amount of data in kilobits per second (kbps) a node transmits, the time interval between the transmission and reception of a packet and the number of packets that are transmitted by a node and fail to reach their destination respectively.

To validate the proposed mechanism's performance and to obtain meaningful statistics, we ran the simulation for each topology about 100 times with different combination of parameter values, so that a confidence level of $95 \%$ is achieved about the validity of our results.

\subsection{Simulation topologies and parameters}

As mentioned earlier, the proposed mechanism has been simulated using six different simulation topologies each with a different number of stations. In order to make the results easy to compare and evaluate, different stations having different traffic were considered as traffic from two distinct applications that exist on the same station contend for the medium in the same way as traffic that exist on two different stations when subjected to differentiated service-based QoS implementations like EDCA. Table 4 shows the distribution of traffic stations for each network topology. The traffic is generated using the built-in NS-2 traffic generation functions with few modifications based on the traffic characteristics of each traffic type. Voice traffic is generated with the parameters of ITU G.711 in order to exercise the proposed mechanism with the heaviest of voice codecs. ITU G.711 codec has $64 \mathrm{kbps}$ of bit rate, 284 bytes of packet size, $2.27 \mu \mathrm{s}$ of packet interval, and $20 \mathrm{~ms}$ of inter-packet interval. Video traffic parameters depend upon the type of browser and the container as mentioned earlier so there are no specific values for video traffic; we took an arbitrary parameter set in confirmation with video traffic models which has $200 \mathrm{kbps}$ of bitrate, 208 bytes of packet size with $3 \mathrm{~s}$ of burst interval and $0.5 \mathrm{~s}$ of idle time interval. Web-browsing and file-sharing traffic is generated using NS-2's built-in 


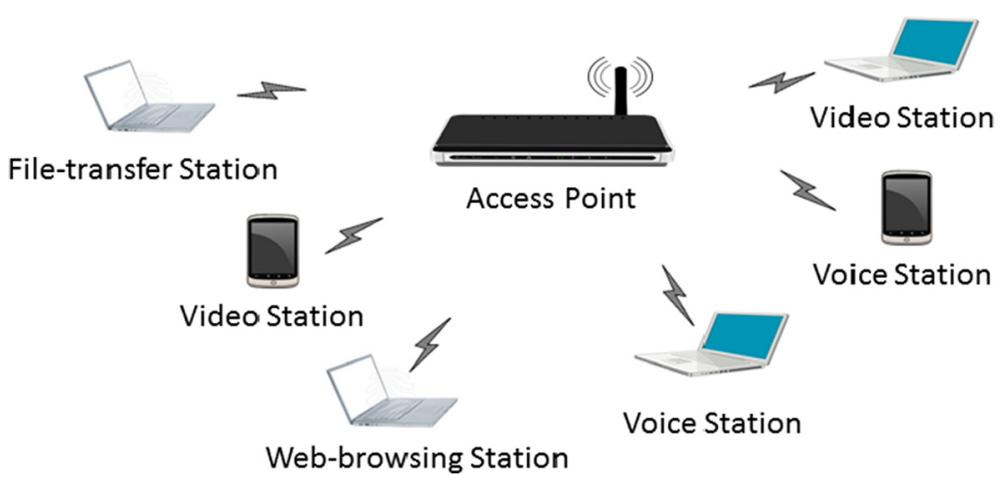

Figure 2 A general WLAN environment exhibiting the 6-station simulation topology.

exponential traffic source with the parameter settings of bit rate, packet interval and packet size in confirmation with the characteristics of file-sharing and web-browsing traffic.

\section{Results and analysis}

We compare the performance of the proposed mechanism with EDCA through three performance metrics of average throughput, average packet delay and average packet loss. Figures 3, 4 and 5 show the average throughput, average packet delay and average packet loss, respectively, as a function of network load (number of transmitting stations) corresponding to the respective network topology.

\subsection{Throughput}

Voice traffic throughput for EDCA and the proposed mechanism closely follow each other, both at the uplink and the downlink when the network load is low (4-5 transmitting stations). However, when the network load is moderate (6-7 transmitting stations), the throughput of the proposed mechanism at the uplink closely follows that of EDCA, but at the downlink, the proposed mechanism faces a slight degradation in throughput as compared to EDCA, this slight depreciation is because of the relatively higher medium occupancy by other applications at that instant. At high network loads (8-9 transmitting stations), sharp depreciation in the throughput of EDCA is seen both at the uplink and downlink while

Table 4 Traffic station distribution for different network topologies

\begin{tabular}{ll}
\hline Network topology & Traffic station distribution (number of stations) \\
\hline 4-station & (1) Voice, (1) video, (1) web-browsing, (1) file-sharing \\
5-station & (2) Voice, (1) video, (1) web-browsing, (1) file-sharing \\
6-station & (2) Voice, (2) video, (1) web-browsing, (1) file-sharing \\
7-station & (2) Voice, (2) video, (2) web-browsing, (1) file-sharing \\
8-station & (2) Voice, (2) video, (2) web-browsing, (2) file-sharing \\
9-station & (3) Voice, (2) video, (2) web-browsing, (2) file-sharing \\
\hline
\end{tabular}

the proposed mechanism offers a gradual depreciation in throughput as depicted in Figure 3a. Video traffic's throughput for EDCA and the proposed mechanism closely follow each other both at the uplink and downlink as shown in Figure 3b. But as the network load increases, abrupt depreciation is experienced by EDCA, and the proposed mechanism betters EDCA at each value of the network load because of the better adaptiveness of the proposed mechanism for video traffic. Webbrowsing traffic observes less throughput at the uplink for the proposed mechanism than EDCA when the network load is low, but when the network load gets higher (6-9 transmitting stations), the proposed mechanism experiences higher throughput than EDCA and the throughput depreciation with increasing network load is also gradual in the case of the proposed mechanism as shown in Figure 3c. At the downlink, the throughput of the web-browsing traffic for the proposed mechanism outperforms EDCA for every value of the network load, conforming to the proposed mechanism's design of awarding more priority to the downlink traffic because of the asymmetric traffic volume at the uplink and the downlink. File-sharing traffic experiences better throughput for the proposed mechanism as compared to EDCA both at the uplink and the downlink as illustrated in Figure 3d. The poor performance of EDCA for nonreal-time traffic supports the argument that EDCA neglects non-real-time applications in favour of real-time applications. The benefits of the proposed mechanism are more prominent at higher network loads but are visible at lower network loads as well. EDCA actually provides overwhelming share of bandwidth to voice traffic which suppresses the throughput of other applications. The proposed mechanism on the other hand provides adequate bandwidth to voice and all other applications. At higher network loads, EDCA is unable to provide such excess of bandwidth to real-time applications either, that is where the performance of the proposed mechanism is maximum because of the better 


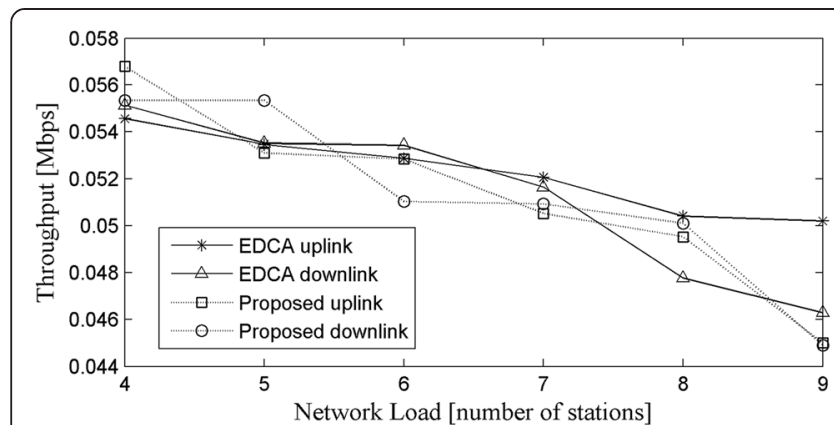

a

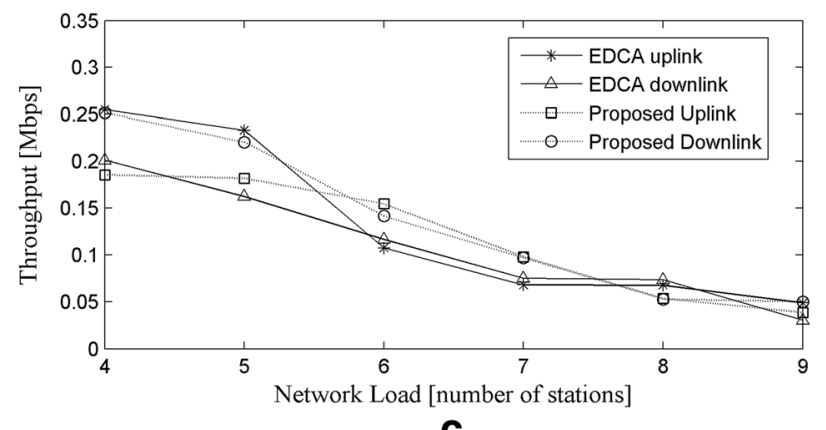

C

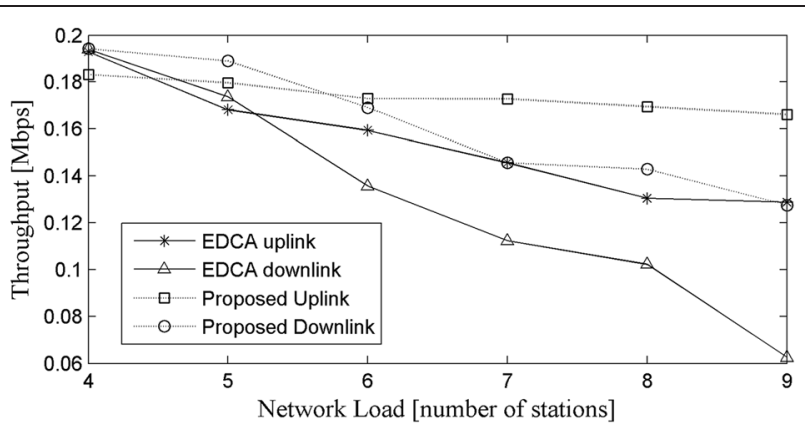

b

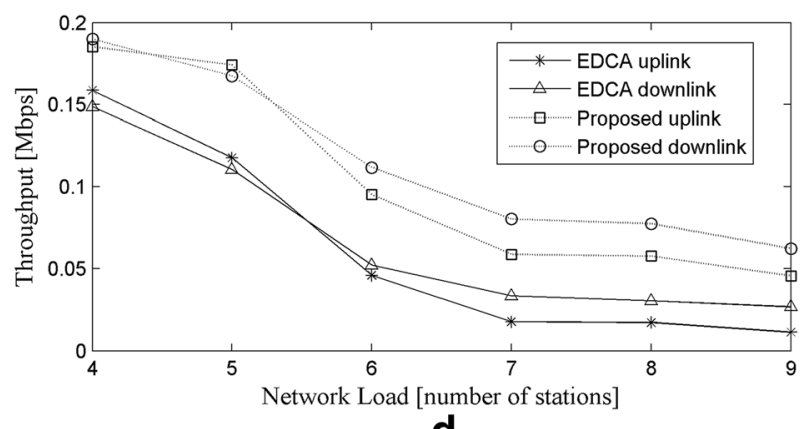

d

Figure 3 Average station throughput over the network load. (a) Voice traffic. (b) Video traffic. (c) Web-browsing traffic. (d) File-sharing traffic.

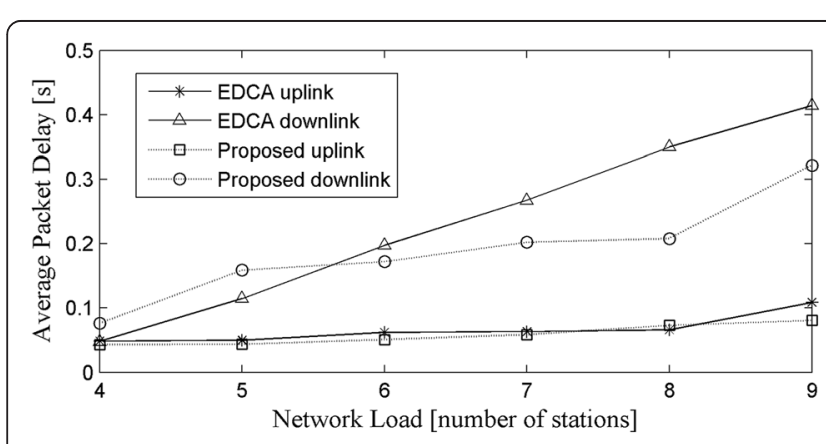

a

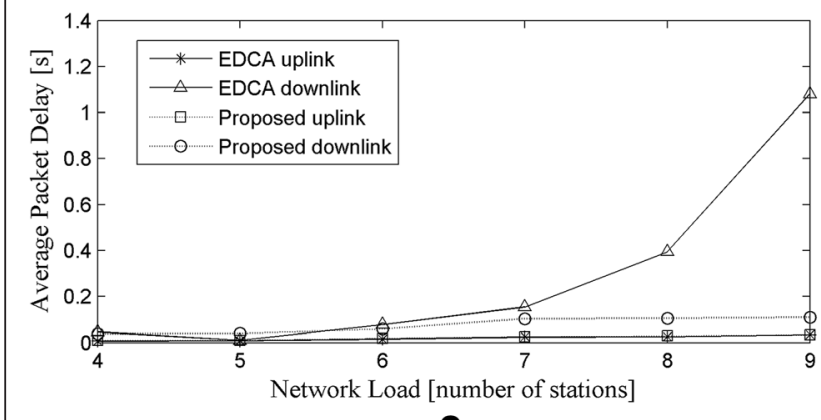

C

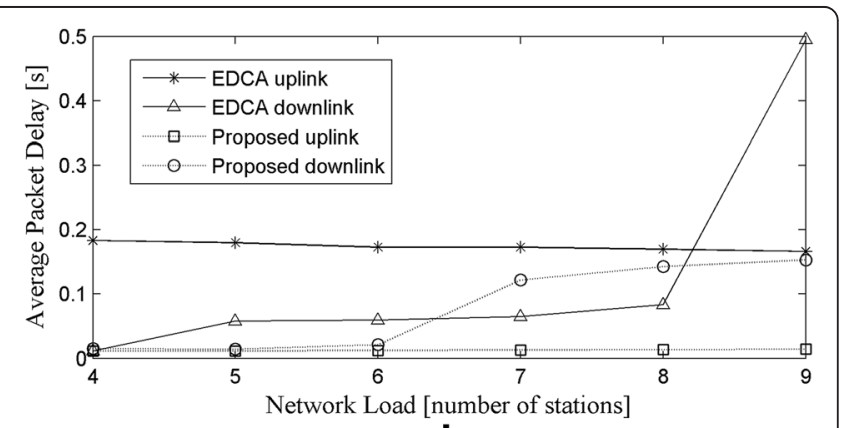

b

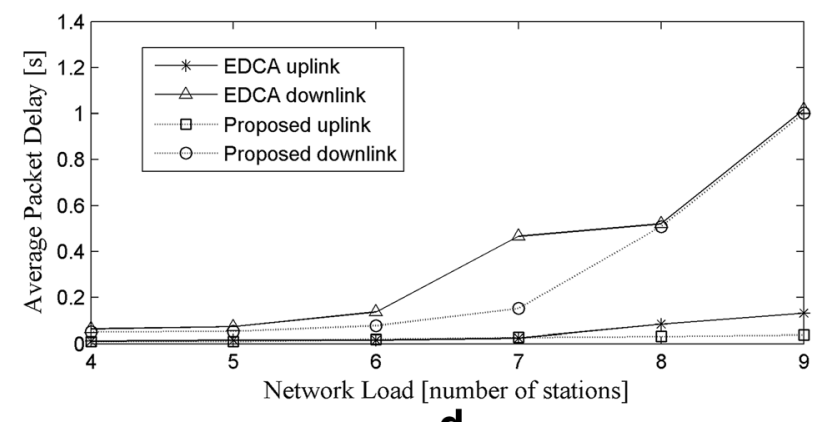

d

Figure 4 Average packet delay as a function of network load. (a) Voice traffic. (b) Video traffic. (c) Web-browsing traffic. (d) File-sharing traffic. 

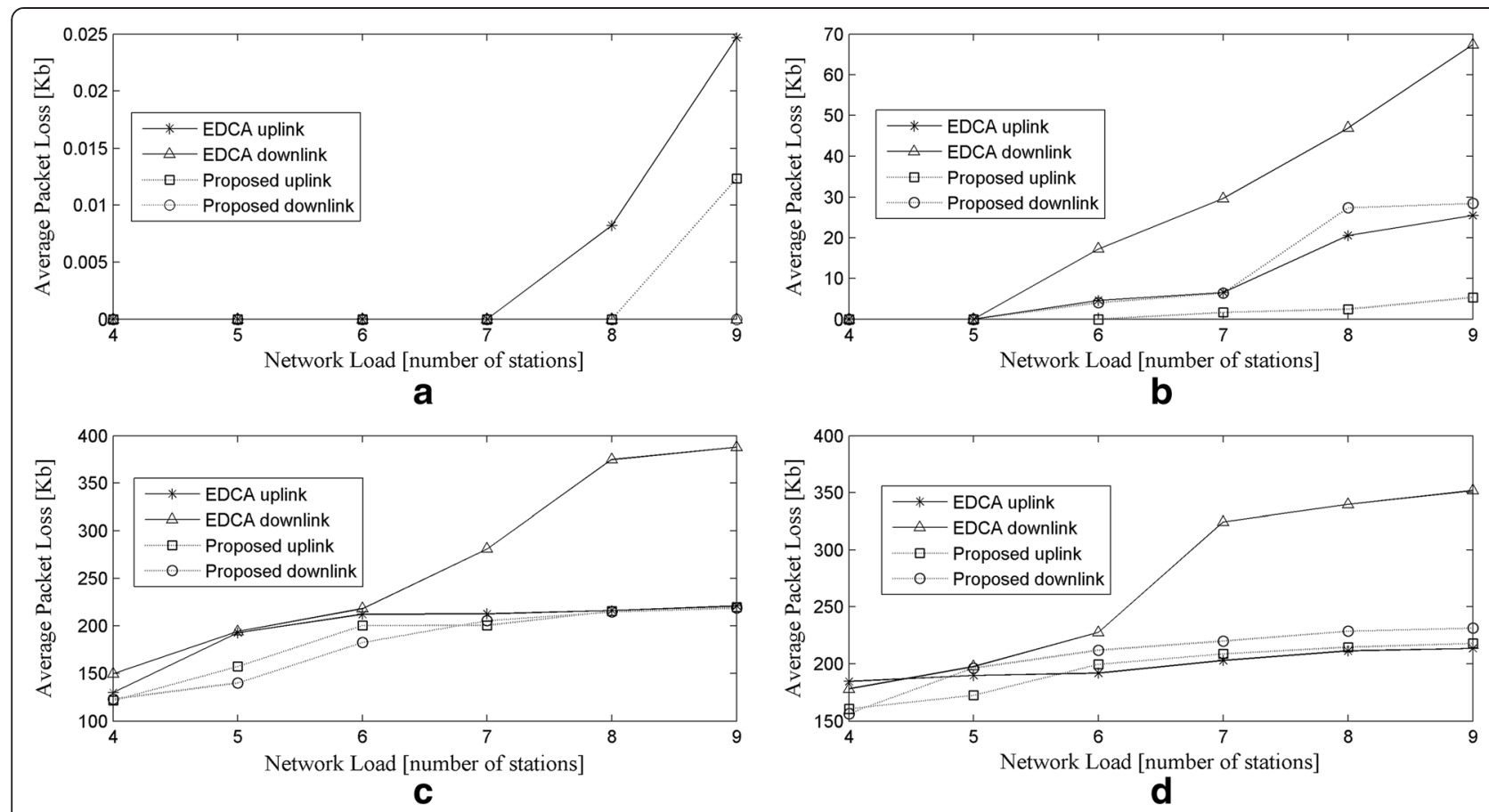

Figure 5 Average packet loss as a function of network load. (a) Voice traffic. (b) Video traffic. (c) Web-browsing traffic. (d) File-sharing traffic.

fairness and more efficient bandwidth utilization features of the proposed mechanism.

\subsection{Packet delay}

Voice traffic's average packet delay is shown in Figure 4a. It can be seen that at the uplink, the performance of EDCA and the proposed mechanism is almost similar, while at the downlink, the proposed mechanism experiences a bit more delay than EDCA; however that delay is still much less than the threshold delay value of $150 \mathrm{~ms}$ recommended in ITU-T G.114 [37]. But as the network load increases, the performance of EDCA degrades drastically while the proposed mechanism allows the increase in delay with the increasing network load in a gradual manner. Video traffic observes less packet delay for the proposed mechanism than EDCA both at the uplink and downlink, but when the network load increases, the delay of the proposed mechanism becomes slightly greater than that of EDCA at the downlink (7-8 transmitting stations). The slight increase in the packet delay of video traffic at moderate network load is due to high medium occupancy/contention by other stations at that instant; in fact, it is an optimization issue and it is well-known that in a set of competing situations, improving one might depreciate others, in other words, the added fairness and overall performance enhancement incorporate some cost as well. However, when the network load is highest (9 transmitting stations), the delay of EDCA jumps to very high values while the packet delay of the proposed mechanism provides comparatively a very gradual increase as shown in Figure $4 \mathrm{~b}$. Webbrowsing traffic experiences almost the same packet delay at the uplink for both EDCA and the proposed mechanism with a slight better performance shown by the proposed mechanism, but at the downlink, the proposed mechanism performs better than EDCA with a good margin as shown in Figure 4c. File-sharing traffic also experiences almost the same packet delay for EDCA and the proposed mechanism both at the uplink and the downlink except for some values at higher network loads where the proposed mechanism offers much less delay than EDCA as illustrated in Figure 4d.

\subsection{Packet loss}

Voice traffic's average packet loss in terms of the amount of transmitted data lost in kilobits for EDCA and the proposed mechanism is shown in Figure 5a. Voice traffic experiences some packet loss at the uplink for the proposed mechanism at higher network loads, but EDCA has much higher packet loss at those network load values. Based on our calculations of packet loss rate for voice traffic by taking the ratio of total amount of data lost with total data sent by the voice station, the packet loss rate for the proposed mechanism at the uplink for 9-station topology comes out to be $0.0005 \%$ while the allowed packet loss rate threshold for voice/ VOIP traffic is $4 \%$ [38], thus complementing the efficiency of the proposed mechanism. For voice traffic, EDCA and the proposed mechanism experience zero packet loss at the downlink. Video traffic packet loss is 
shown in Figure 5b. It is evident that for video traffic, the proposed mechanism minimizes the packet loss both at the uplink and downlink as compared to EDCA. The packet loss threshold of video traffic is believed to be not greater than $0.1 \%$ for best quality playback [38]. Based on our calculations of packet loss rate for video traffic at the 9-station topology by the same method used for the calculation of packet loss rate of voice traffic, the proposed mechanism offers a packet loss rate of $0.0625 \%$ at the uplink. At the downlink, the proposed mechanism offers a packet loss rate of $0.35 \%$ which is slightly greater than the threshold but still much better than EDCA which has a packet loss rate of about $0.85 \%$ for the 9-station topology. Web-browsing traffic's packet loss performance for the proposed mechanism betters EDCA at the uplink and with a great margin at the downlink as shown in Figure 5c. File-sharing traffic observes similar results as at the uplink, the proposed mechanism and EDCA closely follow each other but at the downlink, the proposed mechanism performs better than EDCA with a great margin as evident from Figure $5 d$.

\section{Discussion and performance comparison}

We evaluate the performance of the proposed mechanism by providing the percentage enhancement it offers for throughput, packet delay and packet loss in comparison to EDCA. We discuss our findings as follows.

\subsection{Throughput performance}

Voice traffic throughput has no significant improvement or depreciation at the uplink and at the downlink as voice traffic has low bit rate, due to which all implementations are able to provide the required throughput. Although a slight depreciation in performance can be observed at the downlink for moderate network load but there is no significant anomaly shown by the application in practical as the average throughput drop for the downlink of voice traffic at moderate network load is only $3.92 \%$ and as mentioned in Section 3, the major factors that influence voice traffic performance are packet loss and packet delay. Video traffic on the other hand experiences $5 \%$ to $20 \%$ improvement in throughput at the uplink while it has up to $50 \%$ improvement at the downlink. Web-browsing traffic observes up to $25 \%$ depreciation in throughput at the uplink initially because the proposed mechanism prioritizes medium access of traffic at the downlink as mentioned in Section 4 of the paper, for the reason that the traffic volume of webbrowsing traffic at the uplink is much less than that at the downlink. But later at high network loads, it experiences up to $48 \%$ improvement at the uplink because at high network loads, EDCA could not maintain fairness and overwhelmingly favours real-time applications. At the downlink, web-browsing traffic has up to $40 \%$ enhancement in throughput. File-sharing traffic experiences up to $300 \%$ enhancement in throughput at the uplink while it has up to $150 \%$ enhancement at the downlink with higher improvement in throughput at higher network loads. The throughput comparison between EDCA and the proposed mechanism for every aforementioned application is shown in Figure 6a,b. The high percentage enhancement in throughput for nonreal-time applications like file-sharing explains how EDCA suppresses their throughput by allocating extra medium access to real-time applications even at some of their idle intervals. The proposed mechanism on the other hand provides medium access in a prioritized but efficient manner so that real-time applications get similar or even better performance while non-real-time applications experience far better performance.

\subsection{Packet delay performance}

The packet delay performance of the proposed mechanism for voice, video, web-browsing and file-sharing applications is shown in Figure 7a,b. Voice traffic experiences up to $10 \%$ to $35 \%$ improvement in packet delay performance at the uplink while there is some depreciation in performance as well because the proposed mechanism while maintaining fairness among different applications slightly suppresses the medium access ability of voice applications as compared to EDCA. But the delay for the proposed mechanism is always less than the threshold value as mentioned earlier. At the downlink, there is no significant performance enhancement or depreciation for voice traffic. However, all packet delay values do not exceed the threshold value at the downlink either. Video traffic experiences $5 \%$ to $15 \%$ improvement in packet delay at the uplink, while at the downlink, it has up to $450 \%$ improvement, but with a depreciation of up to $70 \%$ at some points because of high medium occupancy by other stations at those intervals as mentioned earlier. Web-browsing traffic has $5 \%$ to $30 \%$ improvement in packet delay performance at the uplink while it experiences a promising improvement of up to $880 \%$ at the downlink with depreciation of $75 \%$ at a point also due to high relative medium occupancy by other stations at those instants. File-sharing traffic observes $8 \%$ to $72 \%$ improvement in packet delay performance at the uplink, while at the downlink, it has an improvement of $10 \%$ to $70 \%$.

\subsection{Packet loss performance}

Voice traffic experiences up to $100 \%$ enhancement in packet loss performance at high network loads for the uplink, while at the downlink, there is no packet loss both for the EDCA and the proposed mechanism; therefore, no enhancement or depreciation can be seen in Figure 8b. Video traffic observes an encouraging 


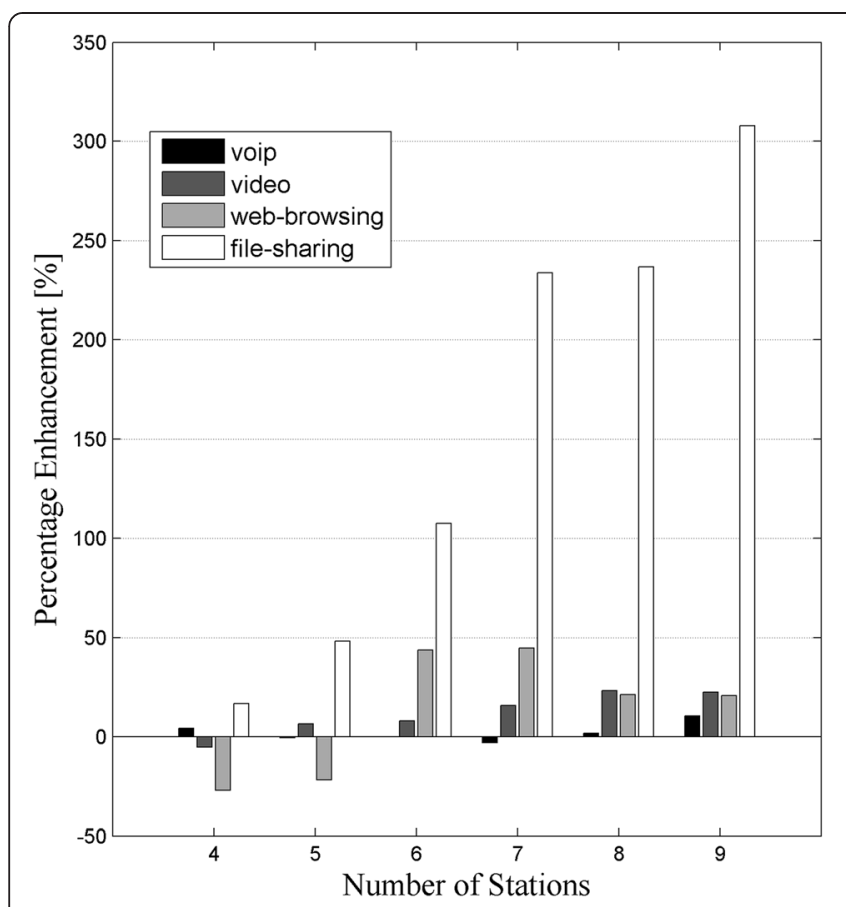

a

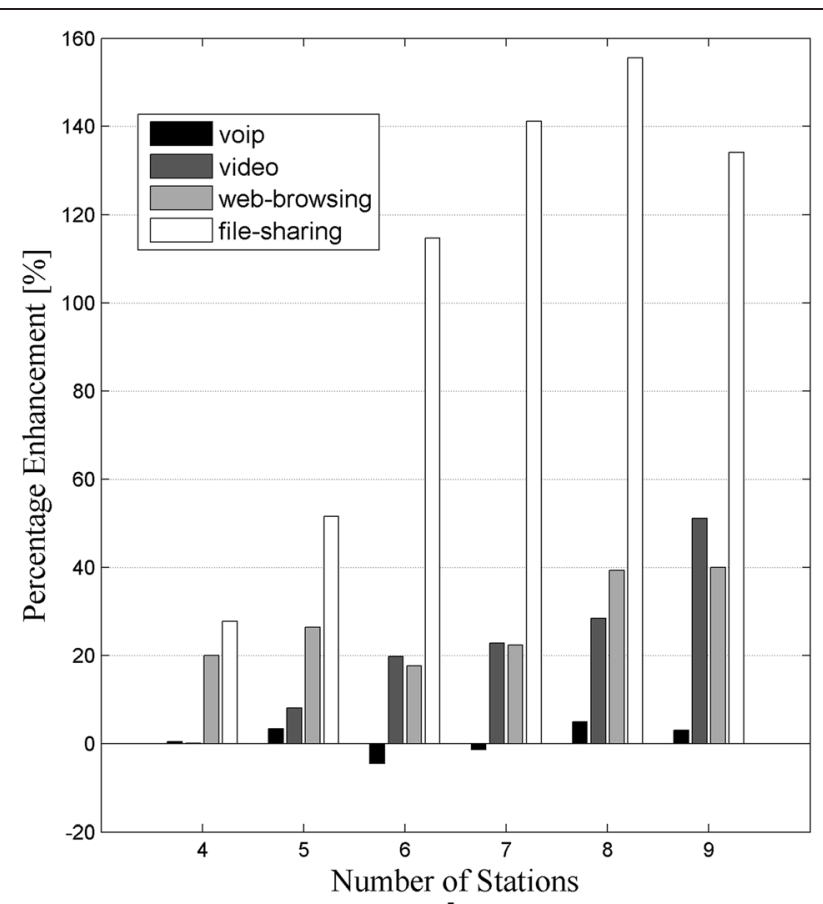

b

Figure 6 Percentage throughput enhancement. (a) Uplink. (b) Downlink.
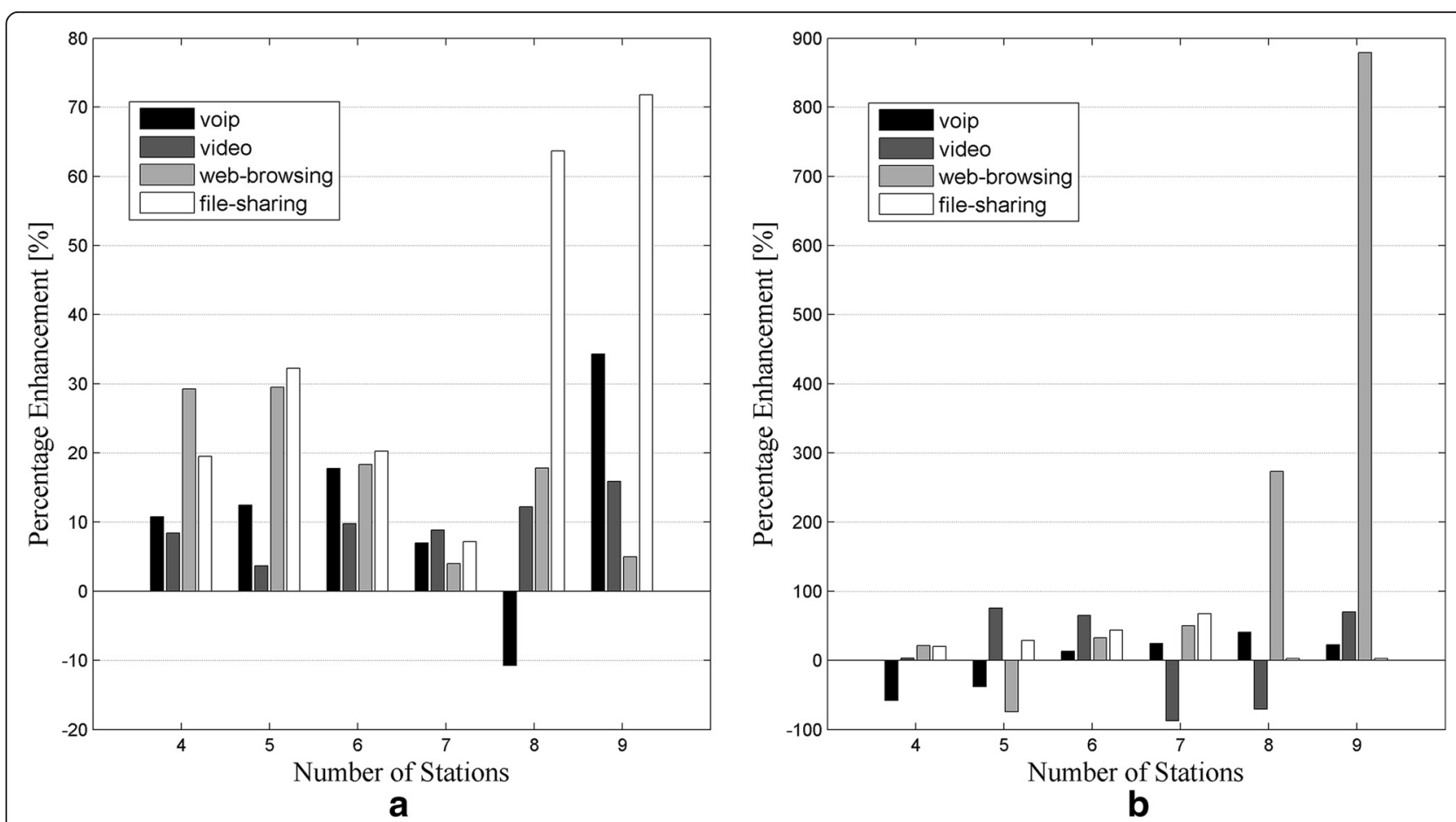

Figure 7 Percentage packet delay enhancement. (a) Uplink. (b) Downlink. 


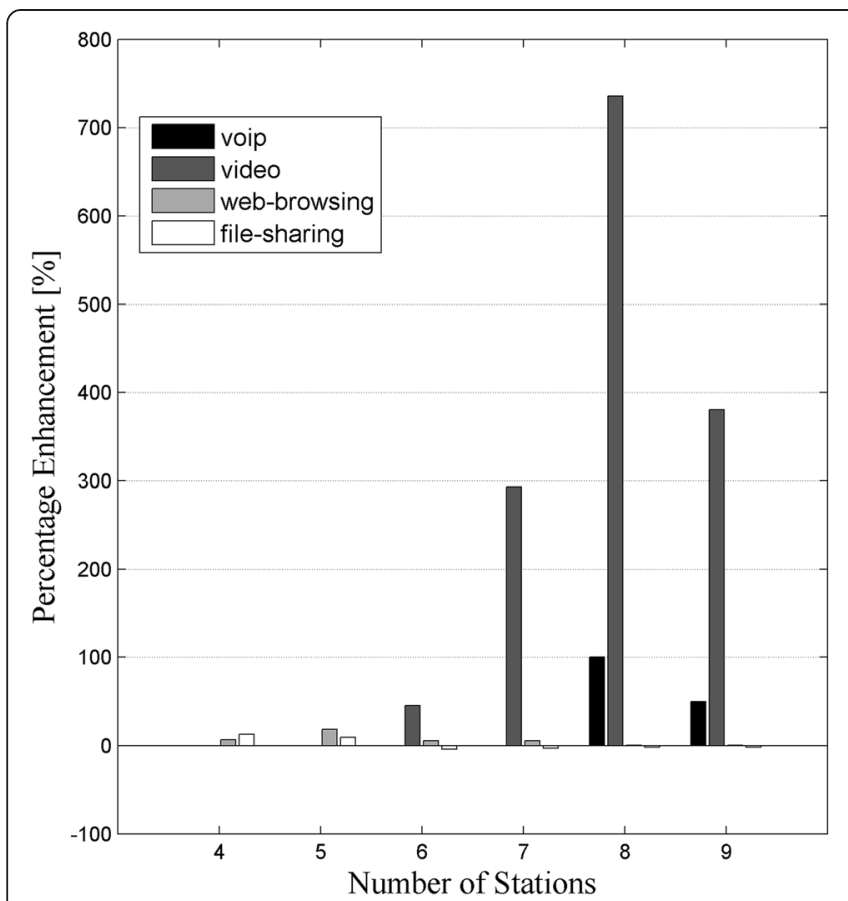

a

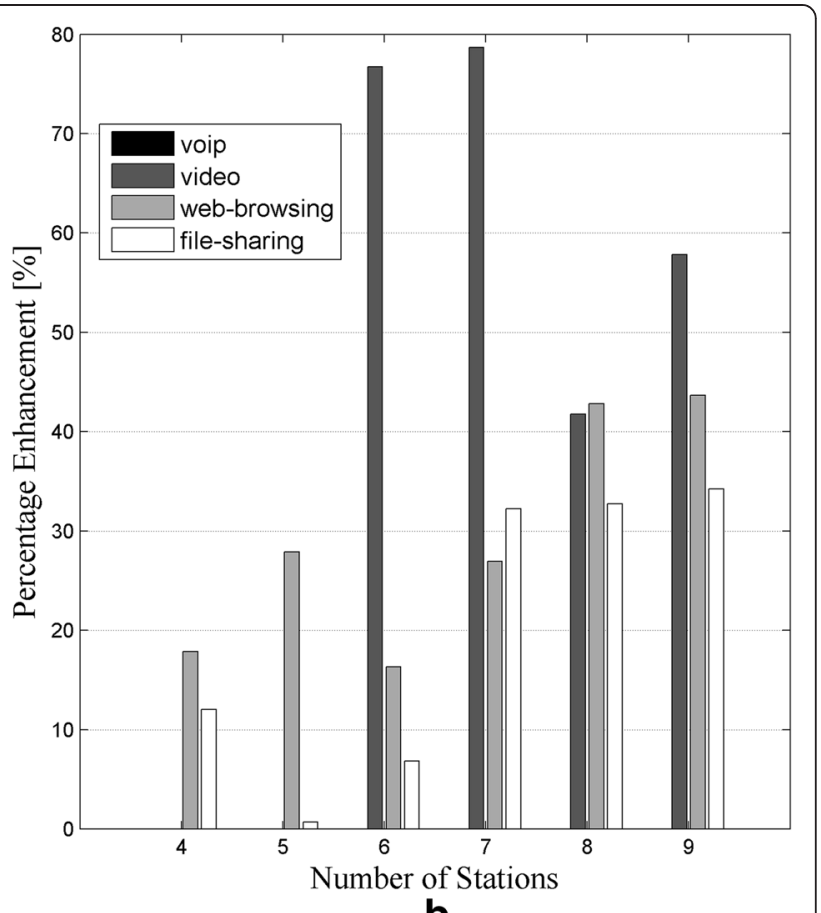

b

Figure 8 Percentage packet loss enhancement. (a) Uplink. (b) Downlink.

improvement in packet loss performance of up to $740 \%$ at the uplink while it experiences an enhancement of $78 \%$ at the downlink. Web-browsing traffic experiences $10 \%$ to $15 \%$ improvement at the uplink while it has up to $43 \%$ improvement in packet loss performance at the downlink. File-sharing has up to $10 \%$ improvement in packet loss performance at the uplink, and at the downlink, it has an enhancement of up to $35 \%$. The percentage packet loss enhancement of the mentioned applications is shown in Figure 8a,b. The packet loss performance of the proposed mechanism is very encouraging as packet losses in addition to adversely affecting the performance of the applications affects the overall network health because when packets are lost, the process of retransmissions for the lost packets is triggered in the already congested network, thus creating more clogging in the network.

\section{Implementation and future work}

Our proposed solution can be implemented on any station by making changes to its wireless adapter's driver and on the access point by adding some functionality in its firmware. The proposed mechanism works with every IEEE 802.11e compatible WLAN protocol. We intend to implement the proposed system on the stations using open source, Linux-based MADWIFI driver [39] and on the access point using DD-WRT, an open source firmware for access points [40]. We have been working with DD-WRT, and some of our future works include the development of the packages that implement our proposed mechanism. Our proposed mechanism can also be implemented using OPENFLOW-enabled switches/ routers [41].

\section{Conclusions}

This paper presents an improved medium access and transmission mechanism for IEEE 802.11 WLANs which ensures provision of QoS for triple play services in a WLAN environment, first, by prioritizing medium access of the stations and the access point according to the volume of the traffic that they have to transmit and according to the sensitivity of the application generating the traffic to packet delay, packet loss and throughput. Secondly, the proposed mechanism makes the transmission style of the nodes adaptive according to the traffic characteristics of the type of traffic a node is about to transmit. Thirdly, for increased transmission efficiency and to ensure fairness among stations and different applications, the proposed mechanism uses the TXOP limit in an adaptive manner according to the traffic behaviour of the traffic the application generates. We have simulated the proposed mechanism in the open source NS-2 simulator and compared the simulation results with the existing EDCA approach being recommended in IEEE $802.11 \mathrm{e}$ draft. The results show considerable improvement in packet loss, packet delay and throughput for inelastic as well as elastic traffic through efficient utilization of the available bandwidth. The research aimed for the provision of QoS for triple play services of 
voice, video and internet access (web-browsing and file-sharing). Accordingly, the proposed scheme provides enhanced services to both real-time applications of voice and video and non-real-time applications of web-browsing and file-sharing by improving fairness and minimizing bandwidth under-utilization. For the provision of minimum required services to real-time applications, EDCA is sufficient, but as the dynamics of internet usage by a general user has changed with the passage of time, simple QoS for only real-time applications solves the problem partially. The achievement of the research and the resulting proposed mechanism on the other hand is the improvement of both real-time and non-real-time application performance and thus enhancement of the overall quality of experience (QoE) of the triple play subscriber.

\section{Competing interests}

The authors declare that they have no competing interests.

\section{Author details}

${ }^{1}$ School of Electrical Engineering and Computer Science (SEECS), National University of Sciences and Technology (NUST), Sector H-12, Islamabad 44000, Pakistan. ${ }^{2}$ College of Computer and Information Systems, Al Yamamah University, Riyadh 11512, Kingdom of Saudi Arabia.

Received: 19 May 2014 Accepted: 22 December 2014

Published online: 24 January 2015

\section{References}

1. Specific Requirements Part 11: Wireless Medium Access Control (MAC) and Physical Layer (PHY) Specifications Amendment 8: MAC Quality of Service (QoS), IEEE Standard 802.11e, 2005

2. T Nilsson and J Farooq, A Novel MAC scheme for solving the QoS parameter adjustment problem in IEEE 802.11e EDCA, in Proc. 2008 IEEE international symposium on a world of wireless, mobile and multimedia networks, Newport Beach, CA, 23-26 June 2008, pp. 1-9

3. Arpit Gupta, Jeongki Min and Injong Rhee, WiFox: Scaling WiFi Performance for Large Audience Environments, in CoNEXT'12 Proceedings of the 8th international conference on Emerging networking experiments and technologies, Cote d'Azur, France, 10-13 December 2012, pp. 217-228

4. W Stallings, Integrated and differentiated services, in High-Speed Networks and Internets: Performance and Quality of Service, ed. by MJ Horton, 2nd edn. (Prentice-Hall, Upper Saddle River, New Jersey, 2002), pp. 469-502

5. Q Qiang, L Jacob, R R Pillai, and B Prabhakaran, MAC protocol enhancements for QOS guarantee and fairness over the IEEE 802.11 wireless LAN, in Proc. International Conference on Computer and Communication Networks, Miami, FL, October 2002

6. N Vaidya, A Dugar, S Gupta, P Bahl, Distributed fair scheduling in a wireless LAN. IEEE Trans. on Mob. Comp 4, 616-629 (2005)

7. C Emma, P Christian, B Christian, K Holger, W Adam, A distributed end-to-end reservation protocol for IEEE 802.11-based wireless mesh networks. IEEE J. on Sel. Areas in Comm 24, 2018-2027 (2006)

8. W Stallings, Protocols for QoS support, in High-Speed Networks and Internets: Performance and Quality of Service, ed. by MJ Horton, 2nd edn. (Prentice-Hall, Upper Saddle River, New Jersey, 2002), pp. 508-521

9. CHR Lin, M Gerla, A synchronous multimedia multihop wireless networks (Proc IEEE INFOCOM, Kobe, Japan, 1997)

10. Yeong-Sheng Chen, Min-Yen Chuang, Fan-Chun Tseng and Chih-Heng Ke, High Performance Distributed Coordination Function with QoS Support in IEEE 802.11e Networks, in Proc. Australasian Telecommunication Networks and Applications Conference (ATNAC), Melbourne, Australia, Nov. 9-11, 2011

11. R Shankar, AT Muthaiya, L Mathew janvier and P Dananjayan, Quality of Service Enhancement for Converging Traffic in EDCA Based IEEE 802.11, in Proc. International Conference on Process Automation, Control and Computing (PACC), Coimbatore, India, 20-22 July 2011
12. T Mishima, S Miyamoto, S Sampei and Wenjie Jiang, Novel DCF-based multi-user MAC protocol and dynamic resource allocation for OFDMA WLAN systems, in Proc. 2013 International Conference on Computing, Networking and Communications (ICNC), San Diego, CA, 28-31 January 2013, pp. 616-620

13. Nan Bao, Junchao Li, Weiwei Xia and Lianfeng Shen, QoS-aware resource allocation algorithm for OFDMA-WLAN integrated system, in Proc. 2013 IEEE Wireless Communications and Networking Conference (WCNC), Shanghai, China, 7-10 April 2013, pp. 807-812

14. M Gatnau Sarret, D Catania, AF Cattoni, JS Ashta and P Mogensen, A multi-QoS aggregation mechanism for improved fairness in WLAN, in Proc. 2013 IEEE $78^{\text {th }}$ Vehicular Technology Conference (VTC Fall), Las Vegas, NV, 2-5 September 2013

15. R Achary, V Vaithiyanathan, P Raj and S Nagarajan, Performance enhancement of IEEE 802.11e WLAN by dynamic adaptive contention window, in Proc. 2014 16 th International Conference on Advanced Communication Technology (ICACT), Pyeongchang, South Korea, 16-19 February 2014, pp. 447-452

16. International Telecommunication Union, Coding of speech at 8kbit/s using conjugate-structure algebraic- code-excited linear prediction (cs-acelp), Telecommunication standardization sector of ITU, Geneva, Switzerland, November 1996

17. International Telecommunication Union, Recommendation G.711, Pulse code modulation (PCM) of voice frequencies (ITU, Geneva, Switzerland, 1988)

18. 3GPP, 3gpp recommendation tr26.075: performance characterization of the AMR speech codec, 1999

19. JF Kurose, KW Ross, Multimedia networking: protocols for real-time interactive applications, in Computer Networking: A Top-Down Approach, 5th edn. (Addison-Wesley, Boston, 2009), pp. 631-636

20. Carlos Ignacio de Mattos, Eduardo Parente Ribeiro, Carlos Marcelo Pedroso, A new model for VolP traffic generation, in The 7th International Telecommunications Symposium (ITS 2010), 2010

21. G. Maier, A Feldmann, V Paxson and M Allman, On dominant characteristics of residential broadband Internet traffic, in 9th ACM SIGCOMM conference on Internet Measurement, 2009, pp. 90-102

22. Adobe Systems Incorporated, Adobe Flash Video File Format Specification, 11.0 edition

23. Microsoft Silverlight, http://www.microsoft.com/silverlight/. Accessed 12 Dec 2013

24. I Hickson, HTML5: a vocabulary and associated APIs for HTML and XHTML, W3C Working, Draftth edn., 2011

25. Global internet phenomena report. Technical report, Sandvine, 2011

26. Ashwin Rao, Arnaud Legout, Yeon-sup Lim, Don Towsley, Chadi Barakat and Walid Dabbous Network characteristics of video streaming traffic, in the Seventh Conference on emerging Networking Experiments and Technologies CoNEXT'11, Tokyo, Japan, 6-9 December 2011

27. T Berners-Lee, R Fielding, and H Frustuk. Hypertext Transfer Protocol—HTTP/1.0. Internet RFC 1945, May 1996

28. R. Fielding, J. Getty, J. Mogul, H. Frystyk, and T. Berners-Lee. Hypertext Transfer Protocol-HTTP/1.1. Internet RFC 2068, July 1997

29. P Badford and M Crovella, Generating representative Web workloads for network and server performance evaluation, in Proc. ACM SIGMETRICS'98, 1998

30. Hyoung-kee Choi and John O. Limb, A behavioral model of Web traffic, in Proc. Seventh International Conference on Network Protocols (ICNP'99), November 1999, pp. 327-334

31. Jeongeun Julie Lee and Maruti Gupta, A new traffic model for current user web browsing user behaviour, (Research @ Intel blog, September 2007), http:// blogs.intel.com/intellabs/2007/09/13/a_new_traffic_model_for_curren/. Accessed 15 Dec 2013

32. J Ishaq, "FTP Traffic Generator", in Technical Report, Department of Electrical Engineering and Computer Science Case Western Reserve University, 10, 2001

33. D Skordoulis, Q Ni, H-H Chen, AP Stephens, C Liu, A Jamalipour, IEEE 802.11n MAC frame aggregation mechanisms for next-generation high-throughput WLANs. IEEE Wireless Comm 15, 40-47 (2008)

34. W Chih-Yu, W Hung-Yu, IEEE 802.11n MAC Enhancement and Performance Evaluation. Mob. Networks and Appl 14,760-771 (2009)

35. Network simulator 2, http://www.isi.edu/nsnam/ns/. Accessed 10 Jan 2013

36. Sven Wiehoelter, Marc Emmelmann, Christian Hoene and Adam Wolisz, TKN EDCA Model for ns-2, (TKN Technical Report TKN-06-003, Berlin, June 2006), http://www2.tkn.tu-berlin.de/research/802.11e_ns2/. Accessed 10 Jan 2013 
37. NG Corporation, Implementing the VOIP Network. Tech. Brief. Series on VOIP and Converged Net. 3(6) (2005)

38. John Williams, Troubleshooting IP Video Quality of Service, http://www.jdsu. com/ProductLiterature/PVIDEOQOS_WP_ACC_TM_AE.pdf. Accessed 15 Dec 2013

39. MadWifi, http://www.madwifi-project.org/. Accessed 27 Jan 2014

40. DD-WRT, http://www.dd-wrt.com/. Accessed 27 Jan 2014

41. OpenFlow, https://www.opennetworking.org/. Accessed 27 Jan 2014

Submit your manuscript to a SpringerOpen ${ }^{\odot}$ journal and benefit from:

- Convenient online submission

- Rigorous peer review

- Immediate publication on acceptance

- Open access: articles freely available online

- High visibility within the field

- Retaining the copyright to your article

Submit your next manuscript at $\boldsymbol{\nabla}$ springeropen.com 